\title{
5' CArG degeneracy in smooth muscle $\alpha$-actin is required for injury-induced gene suppression in vivo
}

\author{
Jennifer A. Hendrix, Brian R. Wamhoff, Oliver G. McDonald, Sanjay Sinha, \\ Tadashi Yoshida, and Gary K. Owens
}

Department of Molecular Physiology and Biological Physics, University of Virginia, Charlottesville, Virginia, USA.

\begin{abstract}
$\mathrm{CC}(\mathrm{A} / \mathrm{T})_{6} \mathrm{GG}$-dependent (CArG-dependent) and serum response factor-dependent (SRF-dependent) mechanisms are required for gene expression in smooth muscle cells (SMCs). However, an unusual feature of many SMC-selective promoter CArG elements is that they contain a conserved single G or C substitution in their central A/T-rich region, which reduces binding affinity for ubiquitously expressed SRF. We hypothesized that this CArG degeneracy contributes to cell-specific expression of smooth muscle $\alpha$-actin in vivo, since substitution of $c$-fos consensus CArGs for the degenerate CArGs resulted in relaxed specificity in cultured cells. Surprisingly, our present results show that these substitutions have no effect on smooth muscle-specific transgene expression during normal development and maturation in transgenic mice. However, these substitutions significantly attenuated injury-induced downregulation of the mutant transgene under conditions where SRF expression was increased but expression of myocardin, a smooth muscle-selective SRF coactivator, was decreased. Finally, chromatin immunoprecipitation analyses, together with cell culture studies, suggested that myocardin selectively enhanced SRF binding to degenerate versus consensus CArG elements. Our results indicate that reductions in myocardin expression and the degeneracy of CArG elements within smooth muscle promoters play a key role in phenotypic switching of smooth muscle cells in vivo, as well as in mediating responses of CArG-dependent smooth muscle genes and growth regulatory genes under conditions in which these 2 classes of genes are differentially expressed.
\end{abstract}

\section{Introduction}

Smooth muscle cells (SMCs) differ from skeletal and cardiac muscle cells in that they retain more extensive plasticity to undergo modulation of their fully mature, contractile phenotype in response to local environmental cues (reviewed in ref. 1). This SMC plasticity is a characteristic feature of SMCs, whether they originated from preexisting SMCs, circulating bone marrow-derived stem cells, or transdifferentiation from other cell types resident in the vessel wall (see discussion in ref. 1). A hallmark of SMC phenotypic modulation is reduced expression of SMC-selective differentiation marker genes, including smooth muscle $\alpha$-actin (SM $\alpha$-actin), SM myosin heavy chain (SMMHC), and SM22. Although there is extensive evidence indicating that phenotypic modulation of fully mature SMCs is associated with the development of several major diseases in humans, including atherosclerosis, hypertension, and cancer (reviewed in ref. 1), a key challenge is to determine the molecular mechanisms that regulate expression of SMC-selective differentiation marker gene expression under physiological and pathophysiological conditions.

$S M \alpha$-actin is the most abundant (2) and earliest-known marker of differentiated SMCs $(3,4)$, and it is a valuable tool for studying differentiation events. Multiple cis elements within the promoter are known to be required for expression of $S M \alpha$-actin in vivo,

Nonstandard abbreviations used: $\mathrm{CArG}, \mathrm{CC}(\mathrm{A} / \mathrm{T})_{6} \mathrm{GG}$; ChIP, chromatin immunoprecipitation; EMSA, electrophoretic mobility shift assay; MHC, myosin heavy chain; MRE, muscle response element; MRTF, myocardin-related transcription factor; Prx1, paired-related homeobox 1; SM, smooth muscle; SMC, smooth muscle cell; SRE, serum response element; SRF, serum response factor; YY1, Yin Yang 1. Conflict of interest: The authors have declared that no conflict of interest exists. including $3 \mathrm{CC}(\mathrm{A} / \mathrm{T})_{6} \mathrm{GG}$ (CArG) elements (5). The CArG element was first identified as part of the serum response element (SRE) contained in the promoter of the immediate early gene $c$-fos, and it is required for serum inducibility of the promoter in response to growth factor stimulation (6). A CArG element is the consensus binding site for serum response factor (SRF), a MADS box transcription factor that binds DNA as a homodimer and promotes the transcription of many CArG-containing, muscle-specific and non-muscle-specific genes $(6,7)$. The question remains as to how a ubiquitously expressed transcription factor such as SRF can regulate 2 seemingly different processes: the activation of musclespecific genes that promote differentiation and the activation of early response genes that promote cell growth and proliferation. The recent discovery of myocardin (8), a cardiac/SM-selective SRF cofactor, has provided valuable insight into this question. Myocardin is required for SMC differentiation $(9,10)$ and can potently transactivate multiple SM marker genes, including $S M \alpha$-actin, in a CArG-dependent manner $(9,11,12)$. However, myocardin fails to activate $c$-fos (8), suggesting that it is part of a regulatory pathway that confers SMC-specific control of CArG/SRF-dependent genes, but not of ubiquitously expressed CArG-containing genes that are involved in the growth response. However, myocardin-induced activation of SMC genes appears to depend on the interaction between myocardin and SRF rather than on direct DNA binding (8), such that an understanding of mechanisms that control SRF binding to the CArG elements is critical to fully understanding the regulation of SMC-selective gene expression.

Interestingly, the promoters of many SMC marker genes, including $S M \alpha$-actin, SMMHC, SM22, desmin, ephrinB2, smoothelin, and calponin, all contain conserved CArG elements (reviewed in ref. 


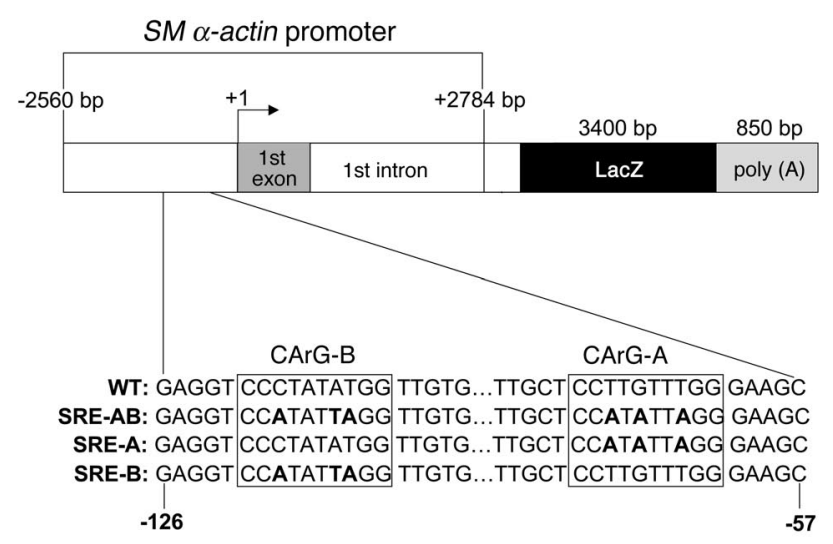

Figure 1

Schematic diagram of wild-type and SRE-substituted mutant/LacZ promoter constructs used to generate transgenic mice. To determine the importance of the reduced SRF binding affinity of the degenerate CArGs for controlling SM $\alpha$-actin expression, we generated mutant LacZ promoter constructs in which a $c$-fos SRE consensus CArG was substituted for the CArG-A and/or CArG-B element(s) within the SM $\alpha$-actin promoter previously shown to mimic expression of the endogenous gene. The resulting mutations are shown in bold.

13), and functional studies have indicated that several of the SM CArG elements are degenerate and exhibit reduced SRF binding affinity as compared to the consensus CArG sequences found in serum response genes such as $c-f o s(7,14)$. For example, both of the SM $\alpha$-actin 5' CArG elements (CArG-A and CArG-B) contain a highly conserved $\mathrm{G}$ or $\mathrm{C}$ substitution within their $\mathrm{A} / \mathrm{T}$-rich cores and exhibit reduced SRF binding affinity in electrophoretic mobility shift assays (EMSAs) as compared to the $c$-fos SRE consensus CArG $(5,7)$. Furthermore, these G/C substitutions have been conserved throughout evolution in all species for which the promoter has been cloned: rat, mouse, chicken, and human (5). The fact that these species are separated by hundreds of millions of years of evolution provides very strong circumstantial evidence that the conserved G/C substitutions play a key role in regulating SMC gene expression. However, at present, there is no direct evidence that the $\mathrm{G} / \mathrm{C}$ substitutions within the CArG elements in any of the SMCselective genes are of functional consequence in vivo.

We hypothesized that there are SMC-selective mechanisms that enhance SRF binding to the degenerate CArGs and that the degeneracy and resulting reduction in SRF binding affinity of the SM $\alpha$-actin 5' CArG elements may be a key mechanism that controls SMC-selective gene expression. In support of this hypothesis, cell culture studies show that substitution of a consensus CArG element (CCATATTAGG) from the $c$-fos SRE into the CArG-A and/or CArG-B site of an $S M \alpha$-actin promoter-reporter construct results in increased $S M \alpha$-actin transcription compared to the wildtype promoter in rat aortic SMCs (7). In addition, high levels of $S M \alpha$-actin transcription have been observed in fibroblasts and endothelial cells, which normally express little to no $S M \alpha$-actin, indicating relaxation of cell-specific expression (7). However, there is no compelling evidence that these results are applicable to regulation of SMC gene expression in vivo. Of note, studies from several labs $(14,15)$ have shown that sequence variations among CArG boxes are important in influencing cell-type specificity of SM22 gene expression under normal developmental conditions in vivo, though this evidence is somewhat controversial.
The primary goal of the present study was to directly assess the role of the degeneracy of SM $\alpha$-actin CArGs in the regulation of SMC-selective gene expression in vivo. To this end, 3 base pair mutations of each of the SM $\alpha$-actin 5' CArGs were generated to convert them to $c$-fos consensus CArGs, and transgenic mice were generated. Transgene expression patterns were determined both under normal conditions and in response to vascular injury. In addition, our present studies address the possible role of this CArG degeneracy, and the SMC-selective SRF coactivator myocardin, in regulating differential expression of CArG-dependent SMC genes and growth regulatory genes.

\section{Results}

Substitution of SM $\alpha$-actin $5^{\prime}$ CArGs with the c-fos SRE consensus CArG had no effect on cell-specific gene expression in vivo. To determine the importance of the reduced SRF binding affinity of CArG-A and CArG-B in controlling cell-specific expression of SM $\alpha$-actin, we generated transgenic animals containing an SRE consensus CArG element substituted for CArG-A (SRE-A), CArG-B (SRE-B), or CArG-A and CArG-B (SRE-AB) in the $S M \alpha$-actin promoter (Figure 1). Our studies employed a 2,560-bp $\alpha$-actin construct plus the first intron $(2,784 \mathrm{bp})$ of the promoter, which mimics expression patterns of the endogenous gene in transgenic mice (5). Surprisingly, in contrast to studies in cultured cells in which these substitutions resulted in relaxed cell specificity (7), substitution of the SRE consensus CArG for either one or both SM $\alpha$-actin 5' CArGs had no detectable effect on cell-specific gene expression across at least 3 independent transgenic founder lines per construct in adult (Figure 2A), E13.5 (data not shown), or E16.5 (Figure 2B) mice. Results of LacZ staining in whole organs, tissues, and embryos were further verified by histological analyses (Figure 2, C and D). Studies indicated complete retention of cell-specific expression patterns of the SRE-substituted mutant transgenes throughout development and maturation. Taken together, these results contradict our initial hypothesis that the SM $\alpha$-actin 5 ' degenerate CArGs are required for cell-specific gene expression and demonstrate that the conservation of degeneracy of the $2 S M \alpha$-actin $5^{\prime}$ CArG elements across multiple species and millions of years of evolution must serve some alternative function.

$S R F$ binding activity of the c-fos SRE consensus CArG was greater than $S R F$ binding activity of the SM $\alpha$-actin $5^{\prime} C A r G$ s in the context of the $S M$ $\alpha$-actin promoter. Based on observations that substitution of the $c$-fos SRE consensus CArG for the SM $\alpha$-actin $5^{\prime}$ CArGs had no detectable effect on SMC specificity in vivo, we questioned whether SRF binding affinity was altered as significantly as would be predicted based on previous EMSA analyses of a wide range of SRE consensus CArG substitution mutants (16-19). EMSAs previously done in our lab showed that the SRE consensus CArG, in the context of the c-fos promoter, bound SRF with greater activity than either CArG-A or CArG-B in the context of the SM $\alpha$-actin promoter (7). However, there was no direct evidence that substitution of the SRE consensus CArG box alone for CArG-A and/or CArG-B in the context of the $S M \alpha$-actin promoter resulted in increased SRF binding activity to the CArG region. In particular, it was not known whether the reduced SRF binding affinity of the $S M \alpha$-actin CArGs, as compared to the SRE consensus CArG, was independent of the G/C substitutions and determined by other sequences within or flanking the $S M$ $\alpha$-actin CArGs. Therefore, we performed EMSA experiments using in vitro translated SRF and a 95-bp SM $\alpha$-actin probe containing both $5^{\prime} \alpha$-actin degenerate CArGs. Competition experiments were 


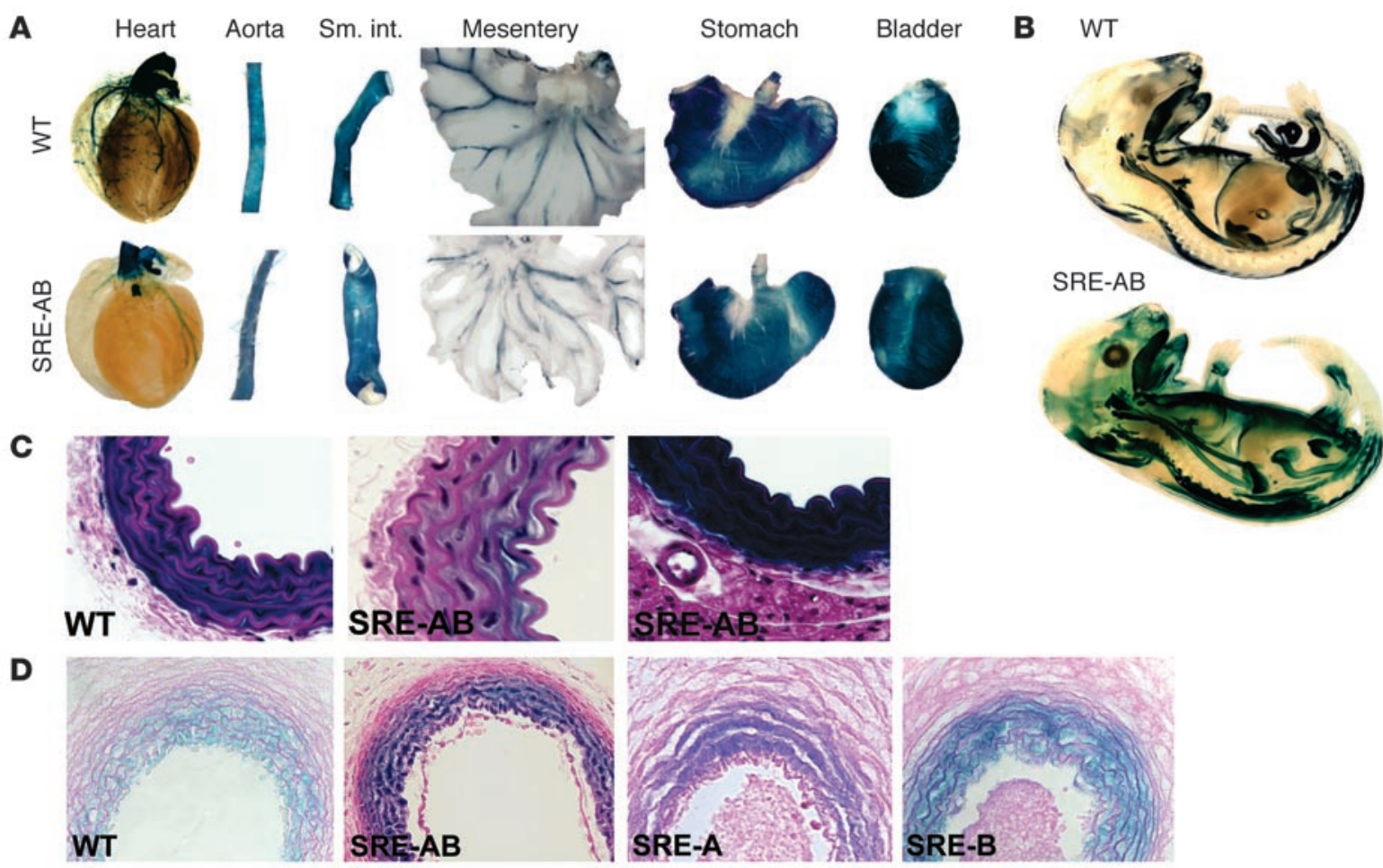

Figure 2

SRE-substituted transgenic mice showed no loss of SMC-specific SM $\alpha$-actin expression during normal development and maturation. (A) Analysis of LacZ expression in whole adult tissues of WT and SRE-AB transgenic mice indicated that the SRE substitutions had no effect on transgene expression in adult tissues. Sm. int., small intestine. (B) Analysis of LacZ expression in E16.5 whole embryos from wild-type and SREAB transgenic mice indicated no loss of specificity upon replacement of SM $\alpha$-actin CArG-A and CArG-B with the $c$-fos SRE consensus CArG. (C) Histological analyses of adult tissues indicated no loss of SMC-specific SM $\alpha$-actin expression in SRE-substituted transgenic mice. Tissues were paraffin-embedded, sectioned, and stained with H\&E. LacZ expression was specific to SMCs in wild-type aorta and in aortas of SRE-AB transgenic mice across multiple independent founder lines. Magnification, $\times 40$. In SRE-substituted transgenic mice, the same LacZ expression pattern was found in all SMC-containing tissues examined, including esophagus and small intestine (data not shown). (D) Histological examination of LacZ- and eosin-stained aortas of E16.5 embryos from wild-type and SRE-substituted transgenic mice indicated no loss of specificity in the mutants. LacZ expression was restricted to the SMC-containing layers of all tissues examined, including the aorta (magnification, $\times 40$ ), esophagus, bronchi, and small intestine (data not shown).

performed with double-stranded, unlabeled wild-type $S M \alpha$-actin, SRE-AB, SRE-A, and SRE-B complexes, all 95 bp in length. Also included in the competition experiments for comparison was a 95-bp, double-stranded, unlabeled c-fos promoter complex, which included the SRE consensus CArG. The SRE-AB, SRE-A, and SRE-B cold competitor complexes competed for SRF binding with the labeled probe more effectively than did the wild-type $S M \alpha$-actin competitor (Figure 3). Not surprisingly, the c-fos DNA complex was the most effective competitor for SRF binding. Importantly, all SRE-substituted mutant DNA complexes competed more effectively than the wild-type competitor did. Therefore, the SRE-substituted $S M \alpha$-actin CArGs exhibited the expected increase in SRF binding affinity, and the lack of an effect of the SRE G/C substitution mutations is thus unlikely to be the consequence of unique sequences within or surrounding the SM $\alpha$-actin CArGs. Our results indicate that increasing the SRF binding affinity of the SM $\alpha$-actin CArGs had no detectable effect on SMC selectivity of the gene, at least during normal development and maturation.

$S R E-A B$ promoter transgene showed an attenuated response compared to the wild-type promoter transgene following injury in mice. The G/C substitutions are completely conserved across all species in which the promoter has been cloned, and they clearly influence SRF binding. Therefore, we hypothesized that the degenerate CArG elements may have evolved to provide a means to differentially regulate CArG-dependent SMC genes compared to CArG-dependent growth response genes under conditions in which SRF is elevated. We further postulated that such a condition might occur following vascular injury, which is known to simultaneously induce CArG/SRF-dependent growth response genes such as $c$-fos (20) and downregulate CArG/SRF-dependent SMC gene expression (21).

Previous studies from our lab have shown that SM marker genes undergo significant transcriptional repression 7 days after vascular injury. In particular, vascular injury studies were done in transgenic mice harboring an $S M \alpha$-actin/LacZ, SM myosin-HC/LacZ, or SM22/ $\mathrm{LacZ}$ transgene. Seven days after vascular injury, all transgenic mice examined showed significantly reduced expression of the $\mathrm{LacZ}$ transgene in the media and developing intima compared to contralateral control vessels. Expression was increased 14 days after injury but was still well below expression levels in the uninjured control artery (21). To determine if the $\mathrm{G} / \mathrm{C}$ substitutions within the $S M \alpha$-actin 5' CArGs played a role in controlling gene expression in response to vascular injury, we performed wire injury studies on at least 5 animals from each of 2 independent SRE-AB founder lines and 2 wild-type $S M \alpha$-actin founder lines. It should be noted 

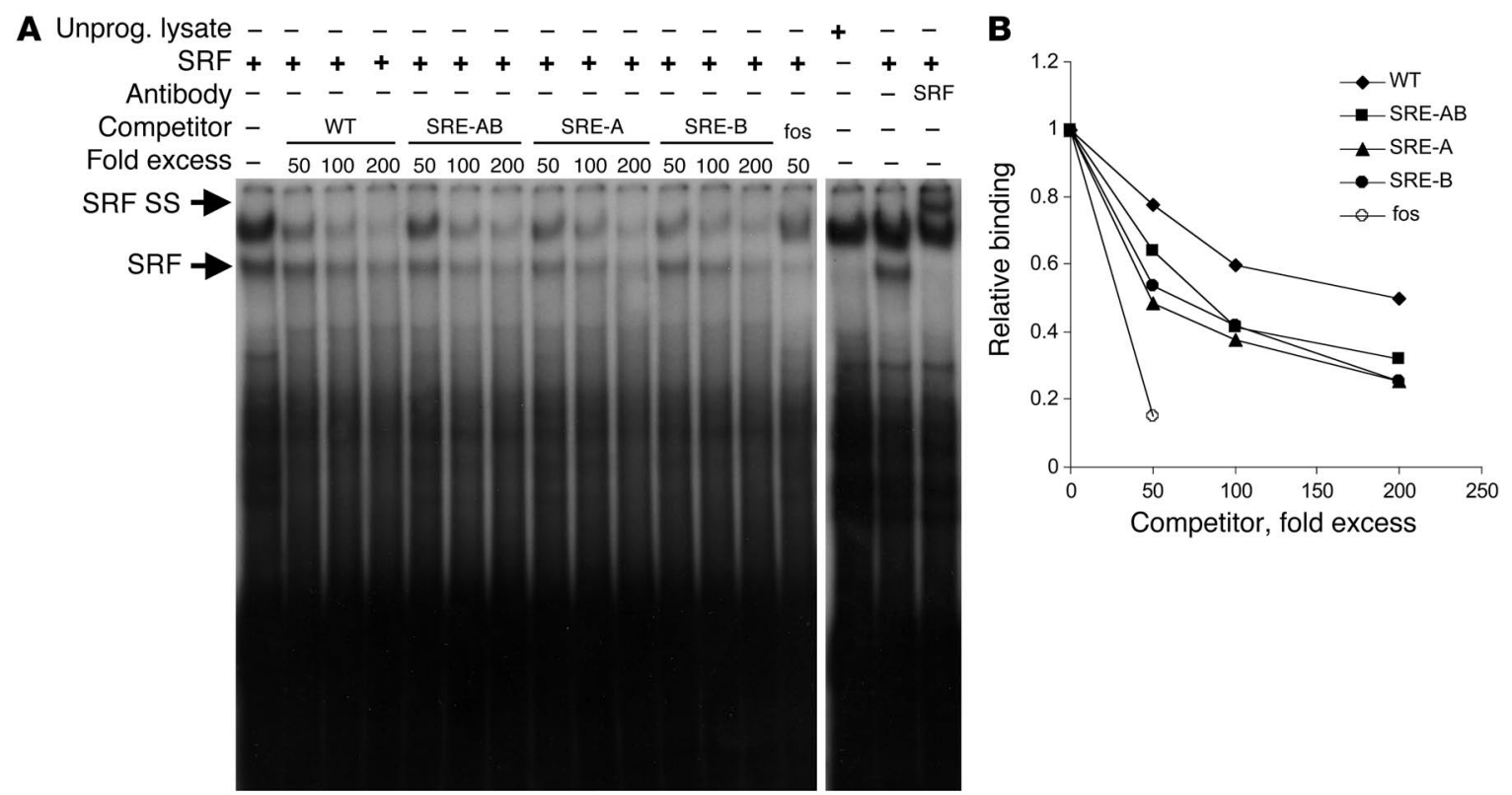

\section{Figure 3}

Oligonucleotides containing the $c$-fos SRE consensus CArG substitution competed for SRF binding activity more effectively than did wild-type $S M \alpha$-actin oligonucleotides (containing CArG-A and/or CArG-B) in EMSAs. (A) In vitro translated SRF and a 95-bp radiolabeled probe harboring the CArG-containing region of the SM $\alpha$-actin promoter were used for EMSAs. Unlabeled 95-bp double-stranded oligonucleotides containing either the SM $\alpha$-actin $5^{\prime}$ CArGs (WT) or the SRE consensus CArG substituted for CArG-A (SRE-A), CArG-B (SRE-B), or CArG-A and CArG-B (SRE-AB) in the context of the SM $\alpha$-actin promoter were used as cold competitors at approximately 50-, 100-, and 200-fold excess over labeled probe. The SRE CArG in the context of the c-fos promoter (fos) was used as a cold competitor at approximately 50-fold excess over labeled probe. The SRF band was supershifted (SRF SS) by the addition of $2 \mu \mathrm{g}$ of anti-SRF rabbit polyclonal antibody. Unprog. lysate, unprogrammed control lysate. (B) Densitometry was performed on the SRF bands (see Figure 5A) and results were plotted relative to maximal SRF binding to the radiolabeled probe in the absence of cold competitor. Results are representative of 3 independent experiments. Statistical analyses were performed using 1-way ANOVA. We found statistically significant differences between percentage of SRF binding in the presence of WT cold competitor and percentage of SRF binding in the presence of SRE-AB, SRE-A, or SRE-B cold competitor under all but 1 condition ( $\times 50$ WT versus $\times 50 \mathrm{SRE}-\mathrm{AB}$ ) across multiple experiments (data not shown).

that since we were assessing a transgene and not the endogenous gene, this approach allowed us to determine pathways that control gene expression within the injured vessel without altering the injury response per se. That is, both our wild-type and SRE-AB transgenic mice contained their normal endogenous $S M \alpha$-actin genes such that there were no differences in function or in the nature of the injury responses between the 2 groups of mice. As such, one can study fundamental mechanisms that control transcription of $S M \alpha$-actin in the context of the normal vascular injury response. Notably, substitution of the SRE consensus CArG for both CArG-A and CArG-B resulted in a significant attenuation in the transgene response to injury. Unlike the wild-type $S M \alpha$-actin gene, which was dramatically downregulated in response to injury, the SRE-AB transgene was expressed in the media and the developing intima of the injured carotid artery at levels equivalent to those seen in the uninjured contralateral control vessel (Figure 4). These results provide evidence that the $\mathrm{G} / \mathrm{C}$ substitutions contained within the $S M$ $\alpha$-actin 5' CArG elements and the resulting reduction in SRF binding affinity are critical for controlling gene expression in response to pathophysiological stimuli such as vascular injury.

Quantitative real-time RT-PCR showed increased levels of SRF expression following vascular injury. To begin to understand possible mechanisms by which substitution of the $S M \alpha$-actin 5' CArGs with the $c$-fos SRE consensus CArG resulted in an attenuated response to vascular injury, we analyzed real-time RT-PCR data from injured rat carotid artery samples to determine the expression pattern of SRF at various time points following injury. SRF levels increased within 1 hour following injury and peaked at 24 hours following injury (Figure 5). At 24 hours, SRF expression levels were approximately 4 times that of the uninjured control and decreased back to control levels by 7 days after injury. These results indicate that, even under circumstances of increased SRF expression, the SM $\alpha$-actin degenerate $C A r G s$ provide a regulatory mechanism that abrogates CArG activation of $S M \alpha$-actin in response to injury. Taken together, our data suggest that SRF can distinguish between muscle-specific and growth factor-specific CArG boxes in vascular injury. Moreover, our results suggest that there may be mechanisms that normally regulate SRF binding to degenerate CArG elements that must be absent or significantly attenuated under conditions of vascular injury. However, it remains to be determined whether this distinction was a result of the CArG sequence alone or whether other factors were involved in helping SRF to distinguish musclespecific from non-muscle-specific CArG elements.

Myocardin increased SRF association with the CArG-containing region of the $S M \alpha$-actin promoter but not of the c-fos promoter in intact chromatin and was downregulated following vascular injury. To assess whether myocardin plays a role in regulating the ability of SRF to distinguish between SM-specific and growth response CArG elements, we used chromatin immunoprecipitation (ChIP) assays to determine the effects of overexpression of myocardin on SRF association with 


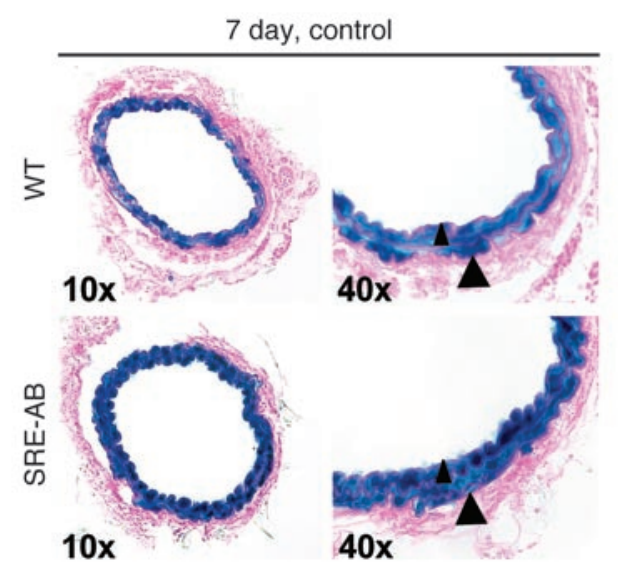

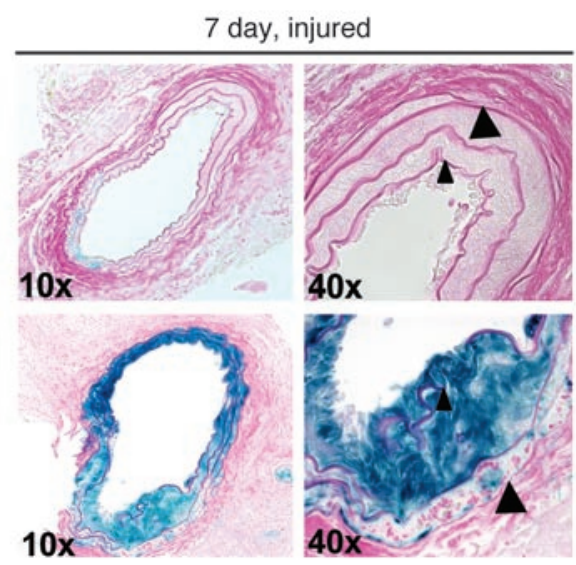

\section{Figure 4}

Analysis of LacZ expression in mouse carotid arteries indicated that the SRE$A B$ transgene is differentially expressed compared with the wild-type SM $\alpha$-actin transgene 7 days following wire injury. Results are representative of at least 5 animals from each of 2 WT and 2 SRE$A B$ independent transgenic founder lines. Small arrowheads indicate the internal elastic lamina, and large arrowheads indicate the external elastic lamina. Magnification is indicated in each panel. the CArG-containing regions of the $S M \alpha$-actin and c-fos promoters. Quantitative real-time PCR analysis of precipitated DNA showed that SRF association was markedly enriched at the SM $\alpha$-actin CArGcontaining region in myocardin-overexpressing cells compared to control cells (Figure 6A). However, there was virtually no difference in SRF binding at the $c$-fos CArG-containing region between cells overexpressing myocardin and control cells. These results suggest that myocardin may contribute to regulation of $S M \alpha$-actin cell-specific gene expression by selectively enhancing SRF binding to the $S M$ $\alpha$-actin degenerate CArGs under normal circumstances. In addition, results suggest that loss of myocardin expression would preferentially decrease $S M \alpha$-actin expression compared to $c$-fos expression.

To determine whether reduced expression of myocardin may contribute to injury-induced suppression of $S M \alpha$-actin expression, we performed quantitative real-time RT-PCR on rat carotid artery samples taken at various time points following vascular injury. Expression of myocardin mRNA was significantly decreased within 3 days following injury compared to the uninjured control, but it returned to control levels by 7 days after injury (Figure 6B). We have previously shown partial reinduction of $S M \alpha$-actin gene expression in injured arteries between 1 week and 3 weeks after injury (21). Although there are no high-quality myocardin antibodies available to confirm these results at the protein level, our results suggest that reduced expression of myocardin may contribute to injury-induced suppression of $S M \alpha$-actin, assuming injury does not result in selective stabilization of myocardin mRNA. Moreover, based on our ChIP assays (Figure 6A), our results also suggest that the reduction in myocardin may selectively decrease SRF binding to the SM $\alpha$-actin degenerate CArGs, but that reduced myocardin has no effect on SRF association with the c-fos CArG. As such, our data support a fundamental mechanism whereby expression of CArGdependent SMC genes may be selectively repressed in response to vascular injury, even in the presence of increased SRF levels. If this is the case, substitution of the $c$-fos SRE consensus CArG for the SM $\alpha$-actin $5^{\prime}$ CArGs would be predicted to decrease myocardin-induced transactivation of $S M \alpha$-actin. Consistent with our hypothesis, cotransfection studies in rat aortic SMCs showed that myocardininduced transactivation of an SRE-substituted SM $\alpha$-actin promoter construct was significantly reduced compared to that of a wild-type promoter construct (Figure 6C). Taken together, these results suggest that myocardin can selectively regulate SRF binding to the SM $\alpha$-actin degenerate CArGs, and loss of myocardin may contribute to repression of $S M \alpha$-actin in response to vascular injury.

\section{Discussion}

The major focus of the studies presented in this report was to identify the biological significance of the highly conserved $S M \alpha$-actin 5 ' degenerate CArG elements. Our in vivo results demonstrated that the $S M \alpha$-actin degenerate CArGs were not required for conferring cell-selective gene expression during normal development. However, when we substituted a $c$-fos consensus CArG with higher SRF-binding affinity for the degenerate CArGs, it resulted in significant attenuation of injury-induced $S M \alpha$-actin downregulation, indicating that the degeneracy of the $S M \alpha$-actin $5^{\prime}$ CArGs is critical for controlling gene expression in response to vascular injury. Of major significance, our results suggest that there is not functional redundancy with respect to pathways that downregulate $S M$ $\alpha$-actin in response to injury - elimination of CArG degeneracy alone rescued gene expression and/or prevented downregulation of $S M \alpha$-actin following injury. That is, a substitution that resulted in a change of only 6 base pairs out of the nearly 5,500 that are required to drive expression of $S M \alpha$-actin in transgenic mice caused a virtual loss of injury-induced suppression of $S M \alpha$-actin. Importantly, no other cis elements previously known to be required for controlling $S M \alpha$-actin gene expression in vivo, such as E-boxes and the TGF- $\beta$ control element (22-24), were disturbed in transgenic mice with the SRE-AB substitution. Taken together, our results indicate that the degenerate CArGs are required for downregulation of $S M \alpha$-actin expression in response to vascular injury.

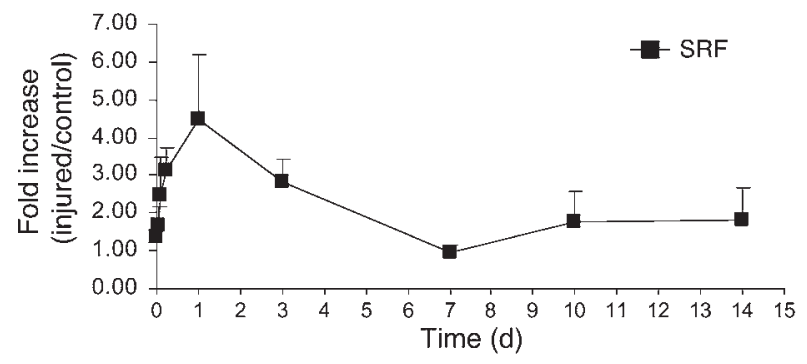

\section{Figure 5}

Temporal expression analysis by real-time RT-PCR of endogenous SRF in balloon-injured carotid arteries showed increased SRF expression following injury. SRF expression was normalized to $18 \mathrm{~S}$ rRNA expression in the injured and uninjured contralateral control vessel. Each time point represents the mean \pm SE of the injured (SRF:18S) vessel normalized to the uninjured (SRF:18S) vessel ( $n=4$ animals per time point). 


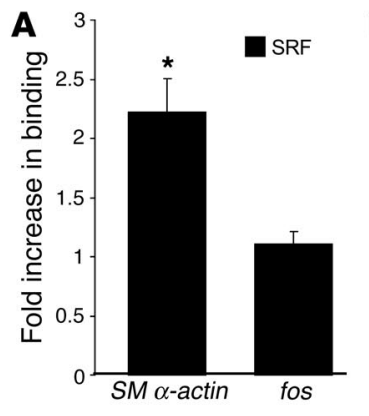

B

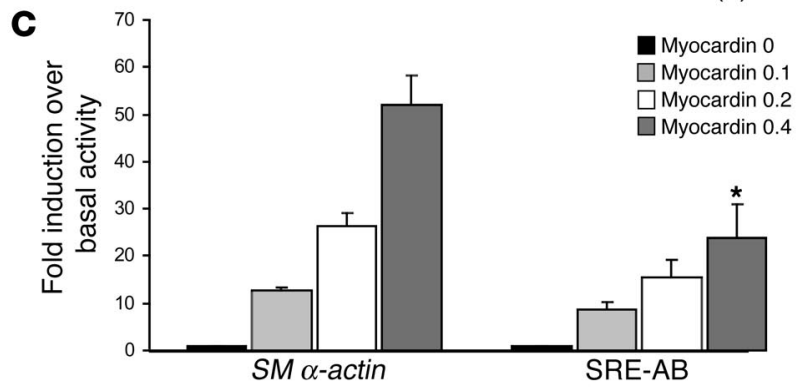

Figure 6

Myocardin can differentially regulate SRF binding to degenerate versus consensus CArGs and is decreased following vascular injury. (A) Results of ChIP assays in cultured rat aortic SMCs indicate that SRF binding is enhanced at the CArG-containing region of the SM $\alpha$-actin promoter but not the $c$-fos promoter in response to myocardin overexpression. Quantitative PCR was used to detect CArG-containing regions of the $S M \alpha$-actin and c-fos promoters in chromatin fragments immunoprecipitated with an SRF antibody. Data represent the mean \pm SE of the fold increase in SRF association in cells overexpressing myocardin versus control cells in 3 independent experiments. A fold increase value of 1 indicates no change in SRF association in cells overexpressing myocardin versus control cells. ${ }^{*} P<0.05$ compared with SRF association at the $c$-fos CArG region under the same conditions. (B) Temporal expression analysis by real-time RT-PCR of endogenous myocardin in balloon-injured rat carotid arteries showed decreased myocardin expression following injury. Myocardin expression was normalized to $18 \mathrm{~S}$ rRNA expression in the injured and uninjured contralateral control vessel. Each time point represents the mean \pm SE of the injured vessel (myocardin:18S) normalized to that of the uninjured (myocardin:18S) vessel $\left(n=4\right.$ animals per time point). ${ }^{\star} P<0.05$ compared with myocardin expression prior to injury. (C) Effect of substitution of CArG-A and CArG-B with the $c$-fos SRE CArG on myocardin responsiveness of $S M \alpha$-actin promoter activity. $S M \alpha$-actin/luciferase and SRE-AB/ luciferase promoter constructs were cotransfected with myocardin into rat aortic SMCs and assayed for luciferase activity. The activity was normalized for protein content. Normalized promoter activities of SM $\alpha$-actin/luciferase and SRE-AB/luciferase in the absence of myocardin were set to 1 . Fold induction over basal promoter activity in response to myocardin was calculated. Values represent the mean \pm SE of 3 independent experiments. ${ }^{*} P<0.05$ compared with WT fold induction under the same conditions.

Previous studies from our lab have shown that there is an additional cis element that is important for mediating downregulation of SM gene expression in response to vascular injury. In particular, Regan et al. (21) showed that mutation of a highly conserved G/Crich region within the $5^{\prime}$ region of the SM22 promoter significantly attenuated injury-induced downregulation of SM22. Interestingly, no such conserved G/C-rich region has been identified in the $S M$ $\alpha$-actin promoter, suggesting that the expression of SMC differentiation marker genes is differentially controlled in response to vascular injury. The mechanisms by which the G/C-repressor region controls SM gene response to injury are not completely understood, but studies have shown that the transcription factors Sp1 and Sp3 may contribute to injury-evoked repression. EMSA studies indicate that Sp1 and Sp3 bind to the conserved G/C-repressor region found in both the SM22 promoter and the SM myosin-HC promoter, and that mutation of these regions abolishes Sp1 and Sp3 binding to the oligonucleotide probes (21). In addition, Sp1 and Sp3 have been shown to be present in the developing intima in vascular injury $(25$, 26). However, there is no direct evidence that these transcription factors contribute to repression of SM marker genes following vascular injury, and it is possible that other factors associate with the G/C-repressor region to regulate gene expression in the face of vascular trauma. Furthermore, mutation of the G/C-repressor region did not completely rescue injury-induced suppression of SM22, thereby suggesting that other factors are involved in control of SM gene repression in response to injury. Of note, similar observations were made in studies done on SRE-AB transgenic mice. Although substitution of the $c$-fos consensus CArG for the $S M \alpha$-actin degenerate CArGs significantly attenuated injury-induced downregulation of the transgene, rescue of gene expression was not complete in all animals. Taken together, these studies suggest that injury-induced changes in gene expression are regulated by multiple factors acting at various cis-regulatory elements whose presence and stoichiometry may be determined by particular pathophysiological events and associated local environmental cues.

Replacement of the SM $\alpha$-actin 5' degenerate CArGs with a $c$-fos consensus CArG resulted in increased SRF binding affinity of the $S M \alpha$-actin promoter in vitro and subsequently attenuated the decrease in the amount of SM $\alpha$-actin promoter transgene following vascular injury in vivo. These results suggest that under normal circumstances, selective mechanisms exist that promote SRF binding to the degenerate CArGs within SMCs, mechanisms that are attenuated in the face of vascular injury. There are a number of potential mechanisms that may be involved in this response. Of major significance, we found that vascular injury was associated with reduced expression of myocardin. Moreover, we demonstrated that overexpression of myocardin in rat aortic SMCs enhanced SRF binding to the SM $\alpha$-actin CArG-containing promoter region, but had virtually no effect on the level of SRF association at the CArG-containing region of the $c$-fos promoter in intact chromatin. Taken together, our results suggest a model in which myocardin selectively enhances SRF binding to the $S M \alpha$-actin degenerate CArG elements and promotes transcription under normal circumstances. However, in response to injury, reduced expression of myocardin and an associated reduction in SRF binding to the degenerate CArGs may result in suppression of gene expression. Interestingly, it appears that the very subtle mutations of the $S M \alpha$-actin 5' degenerate CArGs that enhanced SRF binding affinity were sufficient to rescue expression of the transgene in response to injury, even in the face of reduced levels of myocardin. This suggests that in the presence of CArG ele- 
ments with high SRF-binding affinity, transcriptional expression of $S M \alpha$-actin in response to vascular injury is at least partially myocardin-independent. That is, the SRE-AB transgene may respond to vascular injury similarly to a growth response gene in that it does not require myocardin for enhancement of SRF binding.

In addition to myocardin, a number of other SRF coactivators have been implicated in the control of SMC gene expression, including murine myocardin-related transcription factor A (MRTF-A; also known as MAL/MKL1/BSAC) and MRTF-B (MKL2) $(27,28)$. Of major significance, recent studies by Miralles et al. (29) show that Rho GTPase signaling, which is known to regulate SRF by inducing actin polymerization (30), regulates the subcellular localization of MAL, the human homolog of MRTF-A, in NIH 3T3 fibroblasts. In particular, MAL was found to be localized to the cytoplasm in serum-starved 3T3 cells but accumulated in the nucleus following serum stimulation, and serum-induced translocation of MAL was dependent on Rho-actin signaling. Studies from our lab in cultured rat aortic SMCs showed that inhibition of RhoA with C3 transferase abolished the activity of the SM22 and SM $\alpha$-actin promoter constructs (31). Furthermore, a constitutively active form of RhoA transactivated these promoters, but had no effect on promoters in which the CArGs had been mutated, suggesting that the effects of RhoA on SMC promoter activity are mediated by a CArG/SRF-dependent mechanism. This effect was selective for the SM promoters tested, as the $c$-fos promoter was only modestly affected by overexpression of RhoA. Studies by Camoretti-Mercado and colleagues $(30,32)$ suggest that the RhoA/Rho kinase pathway can alter SMC gene transcription in part by regulating SRF translocation from the nucleus to the cytoplasm. Taken together, these results suggest that the RhoA/Rho kinase pathway may play an important role in controlling cell-specific expression of SM marker genes by regulating SRF and/or MAL subcellular localization, although further studies are needed to fully understand the signaling pathways and the events preceding SM-specific gene transcription. Further studies will also be needed to determine what role, if any, these regulatory processes play in the injury response in vivo.

There are several alternative mechanisms other than regulation of myocardin and/or myocardin-like factors that may contribute to injury-induced repression of $S M \alpha$-actin. For example, we recently demonstrated that siRNA-induced suppression of the homeodomain protein paired-related homeobox 1 (Prx1) in cultured rat aortic SMCs dramatically reduced both basal and angiotensin II-induced activation of SM $\alpha$-actin (33). Moreover, EMSA studies from our lab and others (33-35) have shown that Prx1 can enhance SRF binding to CArG elements. Therefore, we hypothesized that reductions in Prx1 expression may play a key role in injury-induced suppression of $S M \alpha$-actin transcription. Interestingly, real-time RT-PCR analysis of Prx1 levels revealed no detectable change in expression of Prx1 at various time points following vascular injury in rats (data not shown). However, we can not exclude the possibility that changes in Prx1 that are undetectable by real-time RT-PCR or other homeodomain proteins may contribute to control of SM $\alpha$-actin gene expression in response to vascular injury. In addition, members of the Kruppel-like protein family may contribute to regulation of SM genes in response to injury. Specifically, GKLF/ KLF4 has been shown to repress expression of SMC marker genes in cultured cells (24), and thus it will be interesting to determine whether KLF4 can mediate injury-induced suppression of these genes in vivo. Vascular injury may be associated with other regulatory mechanisms such as posttranslational modifications of SRF
$(36,37)$ and alterations in splicing of SRF $(38-41)$ that can influence CArG/SRF binding, and therefore mediate SM gene expression in response to changing environmental cues.

Although the most likely explanation for our observations is that the effects of the SRE-substitution mutations were a direct function of the enhanced SRF binding affinity of the SRE consensus $C A r G$ versus the degenerate $S M \alpha$-actin $C A r G s$, one must also consider the alternative possibility that the degenerate CArGs bind some factor, other than SRF, that normally functions to oppose SRF-induced gene activation in response to injury. For example, Martin et al. (16) showed that Yin Yang 1 (YY1) can compete for binding to the $c$-fos SRE and the skeletal $\alpha$-actin CArG-containing muscle response element (MRE). Furthermore, studies show that binding sites for SRF and YY1 overlap one another and that by inducing a single transversion point mutation at any one of a number of positions within the MRE, YY1 binding can be virtually abolished (16). As such, YY1, or a similar factor, may act as a negative regulator of SRF in SMCs and repress SM gene expression in response to cues such as those elicited by vascular injury. Interestingly, UV cross-linking studies by Strobeck et al. (15) show that YY1 can bind smooth muscle element 4 of the SM22 promoter, although the relevance of this finding is not yet understood. In addition, there may be other unidentified factors that can compete with SRF for CArG binding to repress gene expression, and whose binding may have been altered by the 6-bp change that resulted from substitution of the $c$-fos SRE consensus CArG for the SM $\alpha$-actin 5 ' degenerate CArGs. As such, if a repressor does in fact bind the SMC CArGs, it appears that our mutations may have had dual effects of reducing binding of this hypothetical repressor molecule while simultaneously increasing SRF binding.

We believe the results of the current studies are highly significant in that they have identified a cis element within the SM $\alpha$-actin promoter that appears to be required for suppression of $S M \alpha$-actin in response to vascular injury. Since the promoters of many SMC marker genes characterized to date have conserved degenerate CArG elements (reviewed in ref. 13), it is interesting to speculate that similar regulatory mechanisms may be involved in coordinated suppression of multiple SMC marker genes in response to injury. In addition, since flanking sequences of CArGs are known to influence SRF binding affinity (14), it is possible that the model may be extended to a broader repertoire of genes beyond those predicted based on the presence of base pair substitutions within the 10-bp CArG element itself. It will be of interest to determine whether these regulatory mechanisms also play a role in phenotypic switching of SMCs associated with experimental atherosclerosis. Furthermore, whereas the relative contribution of circulating bone marrow-derived cells (42) and adventitial-derived cells (43) to the developing intima is unresolved and controversial in humans (reviewed in refs. 1, 44), it is intriguing to consider the role these transcriptional regulatory mechanisms may play in lesion investment and differentiation of these cells into SMCs or SM-like cells in response to vascular injury. Finally, although we have presented evidence suggesting that myocardin may play a role in both normal regulation of SMC gene expression and responsiveness to changes in environmental cues (injury in this case), further work is needed to directly test this possibility. Additional studies will also be necessary to determine whether there are other processes that regulate SRF binding to degenerate, low-binding-affinity SMC promoter CArG elements and whether these mechanisms represent a broad mechanism for differential regulation of various classes of CArG-dependent regulatory genes. 


\section{Methods}

Construction of rat SM $\alpha$-actin promoter/LacZ reporters. Constructs used for transgenic studies were generated using PCR-based site-directed mutagenesis. Mutations were made in the context of a -2560 to +2784 bp $S M \alpha$-actin promoter/Lac $Z$ construct as described previously (5), where it was referred to as $\mathrm{p} 2600 \mathrm{Int} / \mathrm{LacZ}$. This construct is often referred to here as WT. The integrity of mutated constructs was verified by DNA sequencing (University of Virginia Sequencing Core). To test the effect of substitution of the $c$-fos consensus CArG in the SM $\alpha$-actin promoter on myocardin responsiveness, HindIII-SalI fragments of the pBL155CAT constructs described previously (7) were blunted and subcloned into the SmaI site of the pGL3-basic vector.

Generation and analysis of transgenic mice. All animal protocols were approved by The University of Virginia Animal Care and Use Committee. Transgenic mice were generated using standard methods $(45,46)$ within the UVA Transgenic Core Facility and analyzed as described previously (5). Briefly, founder lines were established and bred to produce offspring. Transgene presence in embryos was detected by PCR with genomic DNA purified from placenta, and transgene presence in $\mathrm{F}_{1}$ mice was detected by PCR with genomic DNA purified from tail clips. For analysis of transgenic mice at various developmental time points, embryos were harvested at E13.5 or E16.5, fixed with $2 \%$ formaldehyde $/ 0.2 \%$ glutaraldehyde, and stained for LacZ expression with a solution made in PBS containing $5 \mathrm{mM} \mathrm{K}_{3} \mathrm{Fe}(\mathrm{CN})_{6}, 5 \mathrm{mM} \mathrm{K}_{4} \mathrm{Fe}(\mathrm{CN})_{6}$, $2.0 \mathrm{mM} \mathrm{MgCl}_{2}, 1 \mathrm{mg} / \mathrm{ml} \mathrm{X}$-gal (suspended in dimethylformamide), $0.01 \%$ $\mathrm{Na}$-deoxycholate, and $0.2 \% \mathrm{NP}-40$. For transgene analysis in adults, mice that were at least 8 weeks old were perfusion-fixed with $2 \%$ formaldehyde $/ 0.2 \%$ glutaraldehyde, and tissues were excised and stained for LacZ expression. For histological assessment of transgenic mice, embryonic and adult tissues were stained for LacZ expression as described above and processed for routine histology (21). Embryonic sections were stained with eosin and adult sections with hematoxylin and eosin.

Electrophoretic mobility shift assays. EMSAs were performed as previously described (47) with minor modifications, using the following oligonucleotides (only the sense strand is shown): $S M$ $\alpha$-actin, 5'-GAGTTTTGTGCTGAGGTCCCTATATGGTTGTGTTAGAGTGAACGGCCAGCTTCAGCCTGTCTTTGCTCCTTGTTTGGGAAGCGAGTGGGAGGGGA-3'; $S R E-A B ， 5^{\prime}$-GAGTTTTGTGCTGAGGTCCATATTAGGTTGTGTTAGAGTGAACGGCCAGC TTCAGCCTGTCTTTGCTCCATATTAGGGAAGCGAGTGGGAGGGGA-3'; SRE- $A, 5^{\prime}$-GAGTTTTGTGCTGAGGTCCCTATATGGTTGTGTTAGAGTGAACGGCCAGCTTCAGCCTGTCTTTGCTCCATATTAGGGAAGCGAGTGGGAGGGGA-3'; $S R E-B, \quad 5^{\prime}$-GAGTTTTGTGCTGAGG T C CA TA T TA G G T T G T G T TA GA G T GA A C G C C A G C TTCAGCCTGTCTTTGCTCCTTGTTTGGGAAGCGAGTGGGAGGGGA-3'; c-fos, 5'-GCGAGCTGTTCCCGTCAATCCCTCCCTCCTTTACACAGGATGTCCATATTAGGACATCTGCGTCAGGTTTCCACGGCCGGTCCCTGTTGTCCTGG-3'. Complementary oligonucleotides were annealed, gel purified from a $2 \%$ agarose gel using the QIAEX II Gel Extraction Kit (QIAGEN), and the double-stranded duplexes were end-labeled with T4 polynucleotide kinase (New England Biolabs Inc.) and $[\gamma-32 \mathrm{P}]$ ATP (PerkinElmer Inc.). Unincorporated nucleotides were removed using the QIAquick Nucleotide Removal Kit (QIAGEN). SRF was synthesized using the TNT Coupled Reticulocyte Lysate System (Promega Corp.). EMSAs were performed with $20 \mu \mathrm{l}$ of binding reaction that contained doublestranded, end-labeled $S M \alpha$-actin probe (approximately 50,000 cpm), 5\% glycerol, 5 mM HEPES, 35 mM Tris- $\mathrm{HCl}$ (pH 7.5), $200 \mathrm{mM} \mathrm{KCl,} 1.05$ mM EDTA, $1.125 \mathrm{mM}$ dithiothreitol, $4 \mu \mathrm{g}$ poly(dI-dC), $2 \mu \mathrm{l}$ of in vitro synthesized SRF, and cold competitor (approximately 50-, 100-, or 200-fold excess over labeled probe) or antibody $(2 \mu \mathrm{g})$ where indicated. SRF antibody for supershifts was purchased commercially (Santa Cruz Biotechnology Inc.).
The binding reactions were incubated for 30 minutes at room temperature before radiolabeled probe was added, and this was followed by another 30-minute incubation at room temperature. All binding reactions were loaded on a $4.5 \%$ polyacrylamide gel and electrophoresed at $170 \mathrm{~V}$ in $0.5 \times$ TBE. The gels were dried and subjected to autoradiography at $-70^{\circ} \mathrm{C}$.

Vascular injury. Wire carotid vascular injury was performed on 10 - to 12-week-old animals as described previously (21). Injury studies were done on mice from at least 2 independent wild-type and SRE-AB transgenic founder lines to rule out transgene copy number and/or insertional effects. At 7 days following injury, the mice were sacrificed using carbon dioxide asphyxiation and perfused via the left ventricle with sterile PBS. The injured carotid artery (right) and contralateral control artery (left) were harvested, rinsed, and stained for LacZ expression as described previously (5). Carotids were then processed for routine histology and sections $(8 \mu \mathrm{m})$ were counterstained with eosin.

Real-time RT-PCR expression analysis. Injured and uninjured control carotid arteries were harvested from adult rats at various time points following balloon injury. RNA was extracted from the carotids using TRIzol Reagent (Invitrogen Corp.) according to the manufacturer's protocol. One microgram of total RNA from each carotid was reverse-transcribed using Powerscript reverse transcriptase (Clontech) in a $20-\mu \mathrm{l}$ reaction volume. Reactions lacking Powerscript reverse transcriptase were set up in parallel to verify the absence of genomic DNA. Real-time PCR was performed on cDNA samples using primers and a Taqman probe (Integrated DNA Technologies Inc.) designed to amplify the rat SRF or the rat myocardin gene. The sequences of the primers and probe are as follows: SRF forward, 5'-TCTCAGGCACCATCCACCAT-3'; reverse, 5'-CCCAGCTTGCTGTCCTATCAC-3'; SRF probe, 5'-CACAGCCAGGTCCAGGAGCCAGGT-3'; myocardin forward, 5'-AAACCAGGCCCCCTTCC-3'; reverse, 5'-CGGATTCGAAGCTGTTGTCTT-3'; myocardin probe, 5'-ACTCTGACACCTTGAGATCATCCAGGTTTGG-3'. Values for relative SRF or myocardin expression in control and injured arteries were normalized to $18 \mathrm{~S}$ rRNA. The $18 \mathrm{~S}$ normalized injured artery (right carotid) value was then normalized to the contralateral control artery (left carotid) value. Data in Figure 5 and Figure $6 \mathrm{~B}$ represent the mean \pm SE of relative SRF or myocardin mRNA starting quantities of 4 animals per time point.

Quantitative chromatin immunoprecipitation assays. Briefly, rat aortic SMCs were grown in the presence of serum to confluence in $100-\mathrm{mm}$ plates and then treated for 24 hours with 50 MOI of CMV-driven, myocardin-containing adenovirus or $50 \mathrm{MOI}$ of CMV-empty adenovirus (control). Cells were treated with $1 \%$ formaldehyde for 10 minutes at $37^{\circ} \mathrm{C}$ to cross-link protein-DNA and protein-protein interactions within intact chromatin. Chromatin was purified as described previously (48). The cross-linked chromatin was sonicated to shear chromatin fragments of 200-600 base pairs. The sonicated chromatin was immunoprecipitated with $5 \mu \mathrm{l}$ of immunoserum to SRF (Santa Cruz Biotechnology Inc.), while negative control/input DNA was immunoprecipitated with no antibody, and immune complexes were recovered with agarose beads (Upstate Group Inc.). Cross-links were reversed, chromatin was subjected to proteinase $\mathrm{K}$ digestion to remove protein from the DNA, and the DNA was purified via phenol:chloroform extraction. Recovered DNA was quantitated by fluorescence with Picogreen reagent (Molecular Probes Inc.) according to the manufacturer's recommendations. Real-time PCR was performed on genomic DNA from ChIP experiments as described in Litt et al. (49), with minor modifications. Briefly, $1 \mathrm{ng}$ of unknown DNA and $1 \mathrm{ng}$ of input DNA were amplified and the threshold cycle number for linear amplification was determined. Dilutions of input DNA served as the standard curve. The threshold cycle number of the unknown SRF immunoprecipitation sample (IP, $1 \mathrm{ng}$ ) was subtracted from the threshold cycle number of the same amount of reference input sample (Ref, $1 \mathrm{ng}$ ), and then 2 was raised 
to the power of the resulting value. This provided fold enrichment of the target sequence relative to the input, which has a homogenous distribution of target sequence. For any PCR, amplification can be described by the formula $X=X_{\mathrm{o}}(1+E)^{n}$, where $X_{\mathrm{o}}$ is the initial DNA concentration of a target sequence, $X$ is the final DNA concentration of a target sequence, $E$ is the efficiency (a number from 0 to 1 ), and $n$ is the number of cycles. Therefore, if a threshold is the point at which a specific final DNA concentration $X$ is reached, $X$ is a constant, and the number of cycles $\left(C_{t}\right)$ required to reach $X$ is inversely related to the initial target sequence concentration $X_{\mathrm{o}}$. That is, $X_{\mathrm{o}}(\mathrm{IP}) / X_{\mathrm{o}}(\mathrm{Ref})=[X(\mathrm{IP}) / X(\mathrm{Ref})](1+E)^{\mathrm{Ct}(\mathrm{Ref})-\mathrm{Ct}(\mathrm{IP})}$. If $X(\mathrm{Ref})=X(\mathrm{IP})$, which should be true for the same primer set, and $E=1$, then $X_{\mathrm{o}}(\mathrm{IP}) / X_{\mathrm{o}}(\mathrm{Ref})=$ $2^{\mathrm{Ct}(\mathrm{Ref})-\mathrm{Ct}(\mathrm{IP})}$. Therefore, for each primer set, the ratio of IP to Ref can be calculated by subtracting the $C_{\mathrm{t}}$ determined for the target sequence of the IP sample from the $C_{t}$ determined for the target sequence of the reference sample and taking the resulting power of 2 . The data for the no-antibody negative control is then subtracted from the other unknown samples to

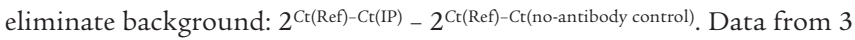
independent experiments were averaged and standard errors of the mean were calculated. Real-time PCR primers were designed to flank the 5' CArG elements of $S M \alpha$-actin and c-fos promoters. This allowed for an accurate quantitative measure of transcription factor binding to the CArG-containing regions of these promoters. Primer sequences were as follows: $S M$ $\alpha$-actin forward, 5'-AGCAGAACAGAGGAATGCAGTGGAAGAGAC-3'; reverse, 5'-CCTCCCACTCGCCTCCCAAACAAGGAGC-3'; c-fos forward, 5'-CGGTTCCCCCCCTGCGCTGCACCCTCAGAG-3'; reverse, 5'-AGAACAACAGGGACCGGCCGTGGAAACCTG-3'. We used 20 pmol of each primer with Sybergreen reagent (Molecular Probes) in real-time PCR reactions. PCR conditions were as follows: 15 -second denaturation at $95^{\circ} \mathrm{C}$, 60 -second annealing at $65^{\circ} \mathrm{C}$, and 45 -second extension at $72^{\circ} \mathrm{C}$ ( 40 cycles).

Transient transfection and luciferase assays. Approximately 24 hours before transfection, rat aortic SMCs were seeded at $1.5 \times 10^{4} \mathrm{cells} / \mathrm{cm}^{2}$ in 12 -well plates. Cells were transiently transfected with plasmid DNA vec- tors using Superfect (QIAGEN) according to the manufacturer's instructions. The total amount of DNA per well was kept constant by adding the corresponding amount of expression vector without a cDNA insert. After transfection, cells were incubated in DMEM supplemented with $10 \%$ fetal calf serum for 48 hours. The Luciferase Assay System (Promega Corp.) was used to harvest cellular lysates. Luminescence and absorbance assays were performed using a Fluostar Optima microplate reader (BMG Labtechnologies Inc.). Luciferase activities were normalized to total protein (Coomassie Plus Protein Assay Reagent, Pierce Biotechnology Inc.). Each sample was examined in duplicate in a single experiment and 3 independent experiments were performed.

Statistical analyses. Statistical analyses were performed using 1-way ANOVA or Student's $t$ test when appropriate. $P$ values of less than 0.05 were considered statistically significant.

\section{Acknowledgments}

This study was supported by NIH grants NIH R01 HL38854, NIH R3757353, and NIH PO1 HL19242 (to G.K. Owens), and by fellowship grant $0215215 \mathrm{U}$ from the Mid-Atlantic Affiliate of the American Heart Association (to J.A. Hendrix). We gratefully acknowledge the useful discussions of M.S. Kumar, C.P. Mack, I. Manabe, C.P. Regan, and the excellent technical assistance of M. Ober, D. Raines, and R. Tripathi.

Received for publication July 7, 2004, and accepted in revised form November 9, 2004.

Address correspondence to: Gary K. Owens, Department of Molecular Physiology and Biological Physics, University of Virginia, 415 Lane Road, MR5 Room 1220, P.O. Box 801394, Charlottesville, Virginia 22908, USA. Phone: (434) 924-9173; Fax: (434) 982-0055; E-mail: gko@virginia.edu.
1. Owens, G.K., Kumar, M.S., and Wamhoff, B.R. 2004. Molecular regulation of vascular smooth muscle cell differentiation in development and disease. Physiol. Rev. 84:767-801.

2. Fatigati, V., and Murphy, R.A. 1984. Actin and tropomyosin variants in smooth muscles. Dependence on tissue type. J. Biol. Chem. 259:14383-14388.

3. Frid, M.G., Shekhonin, B.V., Koteliansky, V.E., and Glukhova, M.A. 1992. Phenotypic changes of human smooth muscle cells during development: late expression of heavy caldesmon and calponin. Dev. Biol. 153:185-193.

4. Hungerford, J.E., Owens, G.K., Argraves, W.S., and Little, C.D. 1996. Development of the aortic vessel wall as defined by vascular smooth muscle and extracellular matrix markers. Dev. Biol. 178:375-392.

5. Mack, C.P., and Owens, G.K. 1999. Regulation of smooth muscle alpha-actin expression in vivo is dependent on CArG elements within the $5^{\prime}$ and first intron promoter regions. Circ. Res. 84:852-861.

6. Treisman, R. 1992. The serum response element. Trends Biochem. Sci. 17:423-426.

7. Hautmann, M.B., Madsen, C.S., Mack, C.P., and Owens, G.K. 1998. Substitution of the degenerate smooth muscle (SM) alpha-actin CC(A/Trich)6GG elements with c-fos serum response elements results in increased basal expression but relaxed SM cell specificity and reduced angiotensin II inducibility. J. Biol. Chem. 273:8398-8406.

8. Wang, D., et al. 2001. Activation of cardiac gene expression by myocardin, a transcriptional cofactor for serum response factor. Cell. 105:851-862.

9. Yoshida, T., et al. 2003. Myocardin is a key regulator of CArG-dependent transcription of multiple smooth muscle marker genes. Circ. Res. 92:856-864.
10. Du, K.L., et al. 2004. Megakaryoblastic leukemia factor-1 (MKL1) transduces cytoskeletal signals and induces smooth muscle cell differentiation from undifferentiated embryonic stem cells. J. Biol. Chem. 279:17578-17586.

11. Chen, J., Kitchen, C.M., Streb, J.W., and Miano, J.M. 2002. Myocardin: a component of a molecular switch for smooth muscle differentiation. J. Mol. Cell. Cardiol. 34:1345-1356.

12. Du, K.L., et al. 2003. Myocardin is a critical serum response factor cofactor in the transcriptional program regulating smooth muscle cell differentiation. Mol. Cell. Biol. 23:2425-2437.

13. Miano, J.M. 2003. Serum response factor: toggling between disparate programs of gene expression. J. Mol. Cell. Cardiol. 35:577-593.

14. Chang, P.S., Li, L., McAnally, J., and Olson, E.N. 2001. Muscle specificity encoded by specific serum response factor-binding sites. J. Biol. Chem. 276:17206-17212.

15. Strobeck, M., et al. 2001. Binding of serum response factor to CArG box sequences is necessary but not sufficient to restrict gene expression to arterial smooth muscle cells. J. Biol. Chem. 276:16418-16424.

16. Martin, K.A., Gualberto, A., Kolman, M.F., Lowry, J., and Walsh, K. 1997. A competitive mechanism of CArG element regulation by YY1 and SRF: implications for assessment of Phox1/MHox transcription factor interactions at CArG elements. DNA Cell Biol. 16:653-661.

17. Santoro, I.M., and Walsh, K. 1991. Natural and synthetic DNA elements with the CArG motif differ in expression and protein-binding properties. Mol. Cell. Biol. 11:6296-6305.
18. Wynne, J., and Treisman, R. 1992. SRF and MCM1 have related but distinct DNA binding specificities. Nucleic Acids Res. 20:3297-3303.

19. Leung, S., and Miyamoto, N.G. 1989. Point mutational analysis of the human $\mathrm{c}$-fos serum response factor binding site. Nucleic Acids Res. 17:1177-1195.

20. Hu, Y., Cheng, L., Hochleitner, B.W., and Xu, Q. 1997. Activation of mitogen-activated protein kinases (ERK/JNK) and AP-1 transcription factor in rat carotid arteries after balloon injury. Arterioscler. Thromb. Vasc. Biol. 17:2808-2816.

21. Regan, C.P., Adam, P.J., Madsen, C.S., and Owens, G.K. 2000. Molecular mechanisms of decreased smooth muscle differentiation marker expression after vascular injury. J. Clin. Invest. 106:1139-1147.

22. Kumar, M.S., Hendrix, J.A., Johnson, A.D., and Owens, G.K. 2003. Smooth muscle alpha-actin gene requires two E-boxes for proper expression in vivo and is a target of class I basic helix-loop-helix proteins. Circ. Res. 92:840-847.

23. Adam, P.J., Regan, C.P., Hautmann, M.B., and Owens, G.K. 2000. Positive- and negative-acting kruppel-like transcription factors bind a transforming growth factor beta control element required for expression of the smooth muscle cell differentiation marker SM22alpha in vivo. J. Biol. Chem. 275:37798-37806.

24. Liu, Y., Sinha, S., and Owens, G. 2003. A transforming growth factor-beta control element required for SM alpha-actin expression in vivo also partially mediates GKLF-dependent transcriptional repression. J. Biol. Chem. 278:48004-48011.

25. Madsen, C.S., Regan, C.P., and Owens, G.K. 1997. Interaction of CArG elements and a GC-rich repressor element in transcriptional regulation 
of the smooth muscle myosin heavy chain gene in vascular smooth muscle cells. J. Biol. Chem. 272:29842-29851.

26. Watanabe, N., et al. 1999. BTEB2, a Kruppellike transcription factor, regulates expression of the SMemb/Nonmuscle myosin heavy chain B (SMemb/NMHC-B) gene. Circ. Res. 85:182-191.

27. Wang, D.Z., et al. 2002. Potentiation of serum response factor activity by a family of myocardinrelated transcription factors. Proc. Natl. Acad. Sci. U. S. A. 99:14855-14860.

28. Cen, B., et al. 2003. Megakaryoblastic leukemia 1 , a potent transcriptional coactivator for serum response factor (SRF), is required for serum induction of SRF target genes. Mol. Cell. Biol. 23:6597-6608.

29. Miralles, F., Posern, G., Zaromytidou, A.I., and Treisman, R. 2003. Actin dynamics control SRF activity by regulation of its coactivator MAL. Cell. 113:329-342.

30. Liu, H.W., et al. 2003. The RhoA/Rho kinase pathway regulates nuclear localization of serum response factor. Am. J. Respir. Cell Mol. Biol. 29:39-47.

31. Mack, C.P., Somlyo, A.V., Hautmann, M., Somlyo, A.P., and Owens, G.K. 2001. Smooth muscle differentiation marker gene expression is regulated by RhoA-mediated actin polymerization. J. Biol. Chem. 276:341-347.

32. Camoretti-Mercado, B., et al. 2000. Physiological control of smooth muscle-specific gene expression through regulated nuclear translocation of serum response factor. J. Biol. Chem. 275:30387-30393.

33. Yoshida, T., Hoofnagle, M.H., and Owens, G.K. 2004. Myocardin and $\operatorname{Prx} 1$ contribute to angiotensin II-induced expression of smooth muscle \{alpha\}-actin. Circ. Res. 94:1075-1082.

34. Hautmann, M.B., Thompson, M.M., Swartz, E.A., Olson, E.N., and Owens, G.K. 1997. Angiotensin II-induced stimulation of smooth muscle alphaactin expression by serum response factor and the homeodomain transcription factor MHox. Circ. Res. 81:600-610.

35. Grueneberg, D.A., Natesan, S., Alexandre, C., and Gilman, M.Z. 1992. Human and Drosophila homeodomain proteins that enhance the DNAbinding activity of serum response factor. Science. 257:1089-1095.

36. Heidenreich, O., et al. 1999. MAPKAP kinase 2 phosphorylates serum response factor in vitro and in vivo. J. Biol. Chem. 274:14434-14443.

37. Matsuzaki, K., et al. 2003. Serum response factor is modulated by the SUMO-1 conjugation system. Biochem. Biophys. Res. Commun. 306:32-38.

38. Vernon, S.M., et al. 1997. Endothelial cell-conditioned medium downregulates smooth muscle contractile protein expression. Am. J. Physiol. 272:C582-C591.

39. Kemp, P.R., and Metcalfe, J.C. 2000. Four isoforms of serum response factor that increase or inhibit smooth-muscle-specific promoter activity. Biochem.J. 345:445-451.

40. Davis, F.J., et al. 2002. Increased expression of alternatively spliced dominant-negative isoform of SRF in human failing hearts. Am. J. Physiol. Heart Circ. Physiol. 282:H1521-H1533.

41. Yang, Y., Beqaj, S., Kemp, P., Ariel, I., and Schuger, L. 2000. Stretch-induced alternative splicing of serum response factor promotes bronchial myo- genesis and is defective in lung hypoplasia. J. Clin. Invest. 106:1321-1330.

42. Han, C.I., Campbell, G.R., and Campbell, J.H. 2001. Circulating bone marrow cells can contribute to neointimal formation. J. Vasc. Res. 38:113-119.

43. Scott, N.A., et al. 1996. Identification of a potential role for the adventitia in vascular lesion formation after balloon overstretch injury of porcine coronary arteries. Circulation. 93:2178-2187.

44. Hoofnagle, M.H., Wamhoff, B.R., and Owens, G.K. 2004. Lost in transdifferentiation. J. Clin. Invest. 113:1249-1251. doi:10.1172/JCI200421761.

45. Li, L., Miano, J.M., Mercer, B., and Olson, E.N. 1996. Expression of the SM22alpha promoter in transgenic mice provides evidence for distinct transcriptional regulatory programs in vascular and visceral smooth muscle cells. J. Cell Biol. 132:849-859.

46. Gordon, J.W., and Ruddle, F.H. 1981. Integration and stable germ line transmission of genes injected into mouse pronuclei. Science. 214:1244-1246.

47. Shimizu, R.T., Blank, R.S., Jervis, R., LawrenzSmith, S.C., and Owens, G.K. 1995. The smooth muscle alpha-actin gene promoter is differentially regulated in smooth muscle versus non-smooth muscle cells. J. Biol. Chem. 270:7631-7643.

48. Manabe, I., and Owens, G.K. 2001. CArG elements control smooth muscle subtype-specific expression of smooth muscle myosin in vivo. J. Clin. Invest. 107:823-834

49. Litt, M.D., Simpson, M., Recillas-Targa, F., Prioleau, M.N., and Felsenfeld, G. 2001. Transitions in histone acetylation reveal boundaries of 3 separately regulated neighboring loci. EMBO J. 20:2224-2235 